Conflict of interest: None declared.

\title{
References
}

1 Kuehni CE, Davis A, Brooke AM, et al. Are all wheezing disorders in very young (preschool) children increasing in prevalence? Lancet 2001; 357: 1821-1825.

2 Spycher BD, Silverman M, Kuehni CE. Phenotypes of childhood asthma: are they real? Clin Exp Allergy 2010; 40: $1130-1141$.

3 Silverman M, Kuehni CE. Early lung development and COPD. Lancet 2007; 370: 717-719.

4 Kuehni CE, Brooke AM, Strippoli MP, et al. Cohort profile: the Leicester respiratory cohorts. Int J Epidemiol 2007; 36: $977-985$

5 Spycher BD, Silverman M, Pescatore AM, et al. Comparison of phenotypes of childhood wheeze and cough in 2 independent cohorts. J Allergy Clin Immunol 2013; 132: 1058-1067.

6 Spycher BD, Silverman M, Brooke AM, et al. Distinguishing phenotypes of childhood wheeze and cough using latent class analysis. Eur Respir J 2008; 31: 974-981.

7 Cai Y, Carty K, Henry RL, et al. Persistence of sputum eosinophilia in children with controlled asthma when compared with healthy children. Eur Respir J 1998; 11: 848-853.

8 Brightling CE. Eosinophils, bronchitis and asthma: pathogenesis of cough and airflow obstruction. Pulm Pharmacol Ther 2011; 24: 324-327.

9 Gaillard EA, Grigg J, Tellabati A, et al. Isolation of cells from the lower airways in infants with wheeze by sputum induction. Eur Respir J 2013; 41: 483-485.

10 Warke TJ, Fitch PS, Brown V, et al. Outgrown asthma does not mean no airways inflammation. Eur Respir J 2002; 19: 284-287.

11 Roorda RJ. Prognostic factors for the outcome of childhood asthma in adolescence. Thorax 1996; 51: Suppl. 1, S7-S12.

12 Pellegrino R, Viegi G, Brusasco V, et al. Interpretative strategies for lung function tests. Eur Respir J 2005; 26 : 948-968.

13 Pavord ID, Pizzichini MM, Pizzichini E, et al. The use of induced sputum to investigate airway inflammation. Thorax 1997; 52: 498-501.

14 Morgan WJ, Stern DA, Sherrill DL, et al. Outcome of asthma and wheezing in the first 6 years of life: follow-up through adolescence. Am J Respir Crit Care Med 2005; 172: 1253-1258.

15 Turner SW, Palmer LJ, Rye PJ, et al. Infants with flow limitation at 4 weeks: outcome at 6 and 11 years. Am J Respir Crit Care Med 2002; 165: 1294-1298.

16 Hovland V, Riiser A, Mowinckel P, et al. The significance of early recurrent wheeze for asthma outcomes in late childhood. Eur Respir J 2013; 41: 838-845.

17 Fleming L, Tsartsali L, Wilson N, et al. Sputum inflammatory phenotypes are not stable in children with asthma. Thorax 2012; 67: 675-681.

18 van den Toorn LM, Overbeek SE, de Jongste JC, et al. Airway inflammation is present during clinical remission of atopic asthma. Am J Respir Crit Care Med 2001; 164: 2107-2113.

\section{Anti-inflammatory effects of targeted lung denervation in patients with COPD}

To the Editor:

Acetylcholine is the primary parasympathetic neurotransmitter in the airways and induces bronchoconstriction via binding with $\mathrm{M}_{3}$ receptors. Cholinergic tone is increased in patients with chronic obstructive pulmonary disease (COPD) and this is the major reversible component of airflow obstruction in the disease [1]. Accordingly, treatment with anticholinergics is an effective bronchodilator therapy for patients with COPD [2]. Recent evidence from animal models of COPD revealed that acetylcholine also promotes airway inflammation and remodelling, which can be inhibited by anticholinergic intervention [3]. Such an anti-inflammatory effect of anticholinergic intervention could be clinically relevant. It has not been previously demonstrated in patients with COPD.

We investigated the effect of targeted lung denervation (TLD) on airway inflammation in COPD. TLD is a novel potential therapy for COPD, in which parasympathetic airway nerves are ablated by applying radiofrequency energy using a bronchoscopically guided catheter-based lung denervation system (Holaira, Inc., Plymouth, MN, USA) [4]. The lung denervation system includes a cooled electrode that is designed to generate therapeutic lesions at a sufficient depth from the inner surface of the main bronchus to ablate the airway nerves that travel parallel to and outside of the main bronchi and into the lungs. An expandable balloon provided protective cooling to minimise airway wall effects in the main bronchi during 
radiofrequency ablation of the nerves. Clinical evidence in COPD suggests that TLD improves lung function and quality of life [4]. We hypothesised that TLD would inhibit airway inflammation. Specifically, we studied whether TLD affects 1) inflammatory cell number and pro-inflammatory cytokine expression in bronchial wash fluid, and 2) gene expression of pro-inflammatory cytokines in bronchial brush specimens.

Patients with moderate-to-severe COPD were recruited as part of a safety and technical feasibility study for TLD (clinical trial number NCT01483534) [4]. Subjects recruited at the University Medical Center Groningen (Groningen, the Netherlands) ( $\mathrm{n}=7$, two males, age $56 \pm 10$ years, smoking history $36 \pm 14$ pack-years, forced expiratory volume in $1 \mathrm{~s}$ (FEV1) $27.6 \pm 6.7 \%$ predicted and $0.70 \pm 0.13 \mathrm{~L}$, forced vital capacity (FVC) $2.19 \pm 0.27 \mathrm{~L}$; all mean \pm SD, pre-bronchodilator FEV1 and FVC) were included in the sub-study investigating the effects of TLD on inflammation. Patients used oral corticosteroids during the interventions, and there was no additional use of anti-inflammatory medication. Patients were allowed to use their regular medication. TLD of the right lung was performed on day 0 . A bronchial wash (two times $25 \mathrm{~mL}$ of saline) and brush were collected from the lung distal to the site of denervation before (day 0) and after denervation (day 30 ). On the bronchial wash, a differential cell count was performed on cytospin preparations stained for MayGrünwald and Giemsa (both from Sigma, St Louis, MO, USA) by counting 400 cells in duplicate in a blinded fashion. Cytokine concentrations in the wash were determined using a multiplex assay (26-plex; Millipore, Billerica, MA, USA). The following cytokines were measured: C-C chemokines CCL2, CCL4 and CCL11, C-X-C chemokines CXCL8 and CXCL10, granulocyte colony-stimulating factor (CSF), interferon (IFN)- $\alpha 2$, interleukin (IL)- 6 and IL-7. The following cytokines were below the detection limit of $3.2 \mathrm{pg} \cdot \mathrm{mL}^{-1}$ : granulocyte-macrophage CSF, IFN- $\gamma$, IL-1 $\alpha$, IL-1 $\beta$, IL-2, IL-3, IL-4, IL-5, IL-10, IL-12 (p40), IL-12 (p70), IL-13, IL-15, IL-17, macrophage inflammatory protein (MIP)-1 $\alpha$, tumour necrosis factor (TNF) $\alpha$ and TNF- $\beta$. From the bronchial brush, total RNA was extracted (Qiagen, Venlo, the Netherlands), reverse transcribed and subjected to quantitative reverse transcriptase PCR to analyse gene expression levels of CXCL8, IL-6, transforming growth factor (TGF)- $\beta$ and mucin MUC5AC using $18 \mathrm{~S}$ as a reference gene. Comparisons between day 0 and day 30 were performed using a Wilcoxon signed rank test and differences were considered statistically significant at $\mathrm{p}<0.05$.

The results are presented in figure 1, where the relative change at day 30 compared with day 0 in levels of neutrophils and protein expression of CXCL8 (IL-8) and CCL4 (MIP-1 $\beta$ ) in the bronchial wash, and the relative change in gene expression of CXCL8, IL-6, TGF- $\beta$ and MUC5AC in the bronchial brush is depicted. For the seven patients studied, the percentage of neutrophils in the bronchial wash was decreased after TLD in five patients, CXCL8 decreased in four patients and CCL4 decreased in six patients $(p=0.047)$. Gene expression of CXCL8 in the brush decreased in six patients $(p=0.031)$, as did IL-6 in five patients, TGF- $\beta$ in six patients $(p=0.047)$ and MUC5AC in five patients. No significant change in the number of macrophages, lymphocytes, eosinophils and the additional cytokines was observed. In this small study population, a repeated measures ANCOVA did not show significant interactions of these parameters with changes in clinical parameters, including FEV1, in response to TLD.

Our findings suggest that TLD attenuates airway inflammation. Evidence from patients on the role of acetylcholine in inflammation is limited. Although the use of tiotropium bromide is associated with a

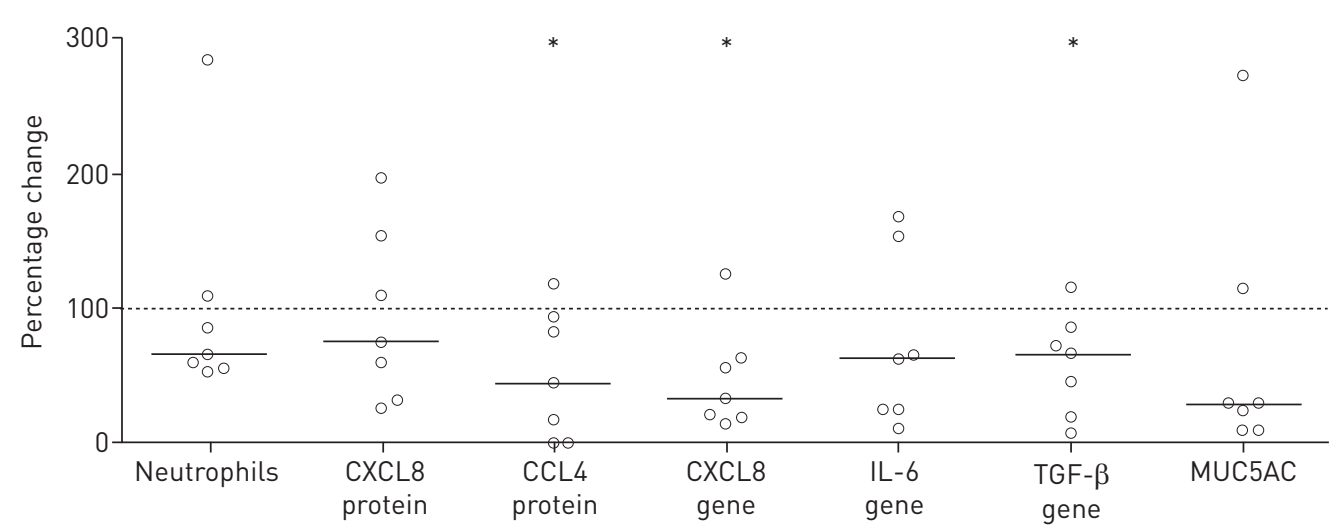

FIGURE 1 Percentage change at day 30 compared with day 0 in levels of neutrophils and protein expression of the C-X-C chemokine CXCL8 (also known as interleukin (IL)-8) and the C-C chemokine CCL4 lalso known as macrophage inflammatory protein-1 $\beta$ ) in bronchial wash, and in gene expression of CXCL8, IL-6, transforming growth factor (TGF)- $\beta$ and mucin MUC5AC in bronchial brush. Baseline values are set to $100 \%$, represented by the dotted line. The horizontal lines represent the median. *: $p<0.05$ (Wilcoxon signed rank test). 
reduction in exacerbation frequency [2], methodological problems have hampered the evaluation of inflammation in the available drug study [5]. This bronchoscopic targeting of the parasympathetic system enabled us to examine the effects on airway inflammation in a completely novel, direct manner. Pre-clinical studies using sheep models have demonstrated effective destruction of cholinergic airway nerves by this device [6]. Although denervation also targets sensory nerve fibres that release pro-inflammatory neuropeptides, such as substance $\mathrm{P}$ and calcitonin gene-related peptide, these nerves have been shown to regenerate in the sheep model, in contrast to cholinergic nerve fibres. We cannot verify the impact of the TLD treatment on individual nerve fibres and mediators in the patients in the current study; therefore, it is important to stress that whereas we believe that these findings suggest a pro-inflammatory role for acetylcholine in the airways, the involvement of additional neurotransmitters and neuropeptides cannot be excluded. In addition, there might be subtle changes in bronchial vessels that could contribute to leukocyte supply, although TLD in sheep did not result in a discernible change in bronchial vessel number or calibre distal to the treatment site. Therefore, we propose that this anti-inflammatory effect of TLD is mediated via inhibition of acetylcholine release.

A pro-inflammatory role for acetylcholine has already been proposed from animal models of COPD [3]. It has been shown that lipopolysaccharide-induced and cigarette smoke-induced inflammation and remodelling can be inhibited by anticholinergic treatment or by knock-out of the muscarinic $\mathrm{M}_{3}$ receptor $[7,8]$.

Acetylcholine is not only a neurotransmitter but is also produced by non-neuronal cells. It has been proposed that this non-neuronal acetylcholine is responsible for the pro-inflammatory effects of acetylcholine $[9,10]$. The current study suggests that neuronal acetylcholine may in fact contribute to inflammation in COPD.

Furthermore, TLD might also have effects on airway remodelling, since a significant reduction in TGF- $\beta$ was observed. This aligns well with the reduction in TGF- $\beta$ we have previously found in muscarinic $\mathrm{M}_{3}$ receptor knock-out mice [8], and with the human data from the UPLIFT (Understanding Potential Long-term Impacts on Function with Tiotropium) trial, where tiotropium reduced lung function decline in a subgroup of patients [11].

There are limitations to this small cohort pilot study, including the limited number of subjects and the lack of control of successful denervation. However, we believe that the findings are promising, and a large-scale multicentre sham-controlled study of the effectiveness of TLD is planned and will include similar analyses. This should provide more evidence for the role of neuronal acetylcholine as a pro-inflammatory mediator. Moreover, it would be interesting to perform similar analyses in patients treated with anticholinergic drugs. Whether anticholinergic intervention will become a tool to treat inflammation is a question to be confirmed in the future.

In conclusion, our findings constitute explorative evidence for a role of TLD in inhibition of inflammation in patients with COPD. This is of clinical interest because current anti-inflammatory therapy, including corticosteroids, is of limited effectiveness. This is a novel and sparsely explored intervention that might affect treatment strategy in COPD in the future. http://ow.ly/QzLbD

Loes E.M. Kistemaker ${ }^{1,2}$, Dirk-Jan Slebos ${ }^{2,3}$, Herman Meurs ${ }^{1,2}$, Huib A.M. Kerstjens ${ }^{2,3}$ and Reinoud Gosens ${ }^{1,2}$

${ }^{1}$ Dept of Molecular Pharmacology, University of Groningen, Groningen, The Netherlands. ${ }^{2}$ GRIAC Research Institute, University of Groningen, University Medical Center Groningen, Groningen, The Netherlands. ${ }^{3}$ University of Groningen, Dept of Respiratory Medicine, University Medical Center Groningen, Groningen, The Netherlands.

Correspondence: Loes E.M. Kistemaker, Dept of Molecular Pharmacology, University of Groningen, A. Deusinglaan 1, 9713 AV Groningen, The Netherlands. E-mail: 1.e.m.kistemaker@rug.nl

Received: March 132015 | Accepted after revision: July 02 2015 | First published online: Sept 042015

Support statement: Financial support was received from the Netherlands Lung Foundation, grant number 3.2.08.014; and from Holaira, Inc., Plymouth, MN, USA.

Conflict of interest: Disclosures can be found alongside the online version of this article at erj.ersjournals.com

\section{References}

1 Gross NJ, Skorodin MS. Role of the parasympathetic system in airway obstruction due to emphysema. $N$ Engl J Med 1984; 311: 421-425.

2 Tashkin DP, Celli B, Senn S, et al. A 4-year trial of tiotropium in chronic obstructive pulmonary disease. $N$ Engl J Med 2008; 359: 1543-1554. 
3 Kistemaker LE, Gosens R. Acetylcholine beyond bronchoconstriction: roles in inflammation and remodeling. Trends Pharmacol Sci 2015; 36: 164-171.

4 Slebos DJ, Klooster K, Koegelenberg CF, et al. Targeted lung denervation for moderate to severe COPD: a pilot study. Thorax 2015; 70: 411-419.

5 Powrie DJ, Wilkinson TM, Donaldson GC, et al. Effect of tiotropium on sputum and serum inflammatory markers and exacerbations in COPD. Eur Respir J 2007; 30: 472-478.

6 Mayse M, Johnson P, Streeter J, et al. Targeted lung denervation in the healthy sheep model - a potential treatment for COPD. Eur Respir J 2014; 44: Suppl. 58, P943.

7 Pera T, Zuidhof A, Valadas J, et al. Tiotropium inhibits pulmonary inflammation and remodelling in a guinea pig model of COPD. Eur Respir J 2011; 38: 789-796.

8 Kistemaker LE, Bos IS, Hylkema MN, et al. Muscarinic receptor subtype-specific effects on cigarette smoke-induced inflammation in mice. Eur Respir J 2013; 42: 1677-1688.

9 Gwilt CR, Donnelly LE, Rogers DF. The non-neuronal cholinergic system in the airways: an unappreciated regulatory role in pulmonary inflammation? Pharmacol Ther 2007; 115: 208-222.

10 Kistemaker LE, Oenema TA, Meurs $\mathrm{H}$, et al. Regulation of airway inflammation and remodeling by muscarinic receptors: perspectives on anticholinergic therapy in asthma and COPD. Life Sci 2012; 91: 1126-1133.

11 Morice AH, Celli B, Kesten S, et al. COPD in young patients: a pre-specified analysis of the four-year trial of tiotropium (UPLIFT). Respir Med 2010; 104: 1659-1667.

\section{Poor agreement between chart-based and objectively identified comorbidities of COPD}

\section{To the Editor:}

Comorbidities contribute significantly to the individual burden of disease in patients with chronic obstructive pulmonary disease (COPD). Their assessment is considered essential for COPD management and is frequently based on data from patients' charts [1]. However, comorbidities are often undiagnosed [2]. Hence, a mismatch between chart-based and objectively identified comorbidities could be anticipated.

The current cross-sectional analyses were based on data collected in the Centre of Expertise for Chronic Organ Failure (CIRO) Comorbidity study (Maastricht University Medical Centre ethics committee approval number 10-3-067). 11 clinically relevant chart-based and objectively identified comorbidities were assessed in a cohort of patients with clinically stable COPD referred for pulmonary rehabilitation. Patients with a history of asthma, $\alpha_{1}$-antitrypsin deficiency, any previous lung surgery, active inflammatory disease, acute myocardial infarction within the last 6 months, any known bone disease other than osteoporosis, current or recently (i.e. 5 years before the study) treated malignant disease, or use of high-dose systemic glucocorticosteroids (>10 mg prednisolone) were excluded.

Comorbidities were identified objectively, based on predefined cut-offs: chronic kidney disease (estimated glomerular filtration rate $<60 \mathrm{~mL} \cdot \mathrm{min}^{-1}$ (Cockroft-Gault formula)) [3]; anaemia (haemoglobin $<8.1 \mathrm{mmol} \cdot \mathrm{L}^{-1}$ in men or $<7.5 \mathrm{mmol} \cdot \mathrm{L}^{-1}$ in women) [4]; hypertension (systolic blood pressure $>140 \mathrm{mmHg}$ or diastolic blood pressure $>90 \mathrm{mmHg}$ ) [5]; low muscle mass (fat-free mass index $<16 \mathrm{~kg} \cdot \mathrm{m}^{-2}$ for men or $<15 \mathrm{~kg} \cdot \mathrm{m}^{-2}$ for women) [6]; diabetes mellitus (fasting plasma glucose $\geqslant 7.0 \mathrm{mmol} \cdot \mathrm{L}^{-1}$ ) [7]; dyslipidaemia (low-density lipoprotein (LDL)-cholesterol $\geqslant 2.5 \mathrm{mmol} \cdot \mathrm{L}^{-1}$ [8]; osteoporosis ( $\mathrm{t}$-score $<-2.5$ ) [9]; symptoms of anxiety and depression (Hospital Anxiety and Depression Scale (HADS) score $\geqslant 10$ ) [10]; atherosclerosis (carotid intima-media thickness $>0.9 \mathrm{~mm}$ ) [5]; and myocardial infarction (cardiac infarction injury score >20) [11]. The technical details of these assessments have been published previously [12].

Chart-based comorbidities were assessed by combining the medical history recorded in the chest physician's referral letter, the patient's interview and an overview of current pharmacological therapy provided by the patient's pharmacist. Chart-based atherosclerosis was defined as ischaemic cerebrovascular disease, coronary artery disease, peripheral arterial disease and/or aortic aneurysms. Concerning pharmacotherapy, the use of angiotensin-converting enzyme inhibitors, angiotensin receptor blockers, calcium channel blockers, $\beta$-blockers or diuretics was labelled as hypertension; oral antidiabetics or insulin as diabetes mellitus; lipid-lowering drugs as dyslipidaemia; bisphosphonates as osteoporosis; anxiolytics or sedatives as anxiety; antidepressants as depression; and platelet aggregation inhibitors (used only for secondary prophylaxis in the Netherlands) [13] as atherosclerosis. Chart-based and objective assessments were independently recorded and assessed. Combined comorbidity was defined by the presence of chart-based and/or objectively identified comorbidity 